Research Article

\title{
ANOVA-Based Magnetic Resonance Imaging in the Diagnosis of Prostate Cancer with Bone Metastasis and Rehabilitation Treatment
}

\author{
Feng Yuan $\mathbb{D}^{1},{ }^{1}$ Ling He $\mathbb{D}^{2},{ }^{2}$ Yancui Zhu $\mathbb{D}^{3},{ }^{3}$ and Honglei Guo $\mathbb{D}^{1}$ \\ ${ }^{1}$ Department of Medical Imaging, The First Affiliated Hospital of Kunming Medical University, Kunming 650032, China \\ ${ }^{2}$ Radiology Department, The First Affiliated Hospital of Yunnan University of Traditional Chinese Medicine, \\ Kunming 650021, China \\ ${ }^{3}$ Intensive Care Unit, The First Affiliated Hospital of Kunming Medical University, Kunming 650032, China \\ Correspondence should be addressed to Honglei Guo; guohonglei@kmmu.edu.cn
}

Received 31 March 2021; Revised 12 May 2021; Accepted 24 May 2021; Published 31 May 2021

Academic Editor: Gustavo Ramirez

Copyright (c) 2021 Feng Yuan et al. This is an open access article distributed under the Creative Commons Attribution License, which permits unrestricted use, distribution, and reproduction in any medium, provided the original work is properly cited.

\begin{abstract}
This study was to analyze the magnetic resonance imaging (MRI) characteristics of prostate cancer patients with bone metastasis and the difference in the rehabilitation effect of patients with different bone metastases. The MRI, diffusion weighted imaging (DWI), magnetic resonance elastic imaging (MRE), and MRI + DWI + MRE imaging were performed on 200 prostate cancer patients in the First Affiliated Hospital of Kunming Medical University hospital. The prostate-specific membrane antigen (PSMA), prostate-specific antigen (PSA), and prostate volume in patients with bone metastases were analyzed. The sensitivity, specificity, and accuracy of the four detection methods of bone metastases in prostate cancer were detected. In addition, the changes of the bone metabolism index $\beta$-special collagen sequence $(\beta$-CTX), type I procollagen amino terminal propeptide (PINP), and osteocalcin (BGP) of the patients were analyzed and compared before and after the treatment. The results showed that the levels of PSMA4 $(17.35 \pm 51.64 \mathrm{ng} / \mathrm{mL})$ and PSA $(15.86 \pm 6.33 \mathrm{ng} / \mathrm{mL})$ in nonbone metastatic prostate cancer patients were much lower than those of osteogenic bone metastases $(668.95 \pm 47.13 \mathrm{ng} / \mathrm{mL}$ and $202.15 \pm 31.53 \mathrm{ng} / \mathrm{mL})$, mixed bone metastases $(637.63 \pm 41.35 \mathrm{ng} / \mathrm{mL}$ and $186.45 \pm 24.86 \mathrm{ng} / \mathrm{mL})$ prostate patients. The sensitivity $(96.25 \%)$, specificity $(89.85 \%)$, and accuracy (98.53\%) of MRI + DWI + MRE in the prostate cancer bone metastasis were obviously higher than those of MRI (86.46\%, 78.31\%, and $90.31 \%)$, DWI $(88.11 \%, 82.53 \%$, and $91.43 \%)$, and MRE (83.36\%, 76.94\%, and 89.76\%). The levels of $\beta$-CTX (0.41 $\pm 0.07 \mathrm{ng} /$ $\mathrm{mL})$, PINP $(39.04 \pm 6.38 \mathrm{ng} / \mathrm{mL})$, and BGP $(17.56 \pm 4.22 \mathrm{ng} / \mathrm{mL})$ after treatment in patients with nonbone metastasis prostate cancer were greatly lower than those of osteogenic bone metastases $(0.66 \pm 0.08 \mathrm{ng} / \mathrm{mL}, 51.45 \pm 7.45 \mathrm{ng} / \mathrm{mL}$, and $33.65 \pm 6.14 \mathrm{ng} /$ $\mathrm{mL})$ and patients with mixed bone metastases $(0.75 \pm 0.12 \mathrm{ng} / \mathrm{mL}, 53.66 \pm 9.22 \mathrm{ng} / \mathrm{mL}$, and $31.24 \pm 5.73 \mathrm{ng} / \mathrm{mL})$, showing statistically obvious differences $(P<0.05)$. In short, the sensitivity, specificity, and accuracy of MRI + DWI + MRE in detection of prostate cancer bone metastasis were better than those of a single examination method, showing significant impacts on the rehabilitation of patients with prostate cancer bone metastasis.
\end{abstract}

\section{Introduction}

In Europe and the United States, the case fatality rate of prostate cancer is second only to that of lung cancer, which ranks first. Its cancer tissue cells have a higher density and their spreading ability is greatly reduced. It is one of the more common malignant tumors in the male genitourinary system [1]. The first symptom of some prostate cancer patients is bone pain. Bone metastasis is found first, and it is the most likely to occur. The disease course is long, the site is hidden, the diagnosis is difficult, the differences between individuals are large, and most of them are found in the late course of the disease, so that the best time to treat is missed [2]. Studies have shown that the mortality rate of prostate cancer patients with bone metastasis is extremely high; it has leapt to the third place among male genitourinary system 
malignancies in China, and its harm far exceeds the prostate cancer itself $[3,4]$. In order to maximize the survival rate and prognosis of patients, it is necessary to determine the clinical stage of the disease course, so there must be a reasonable treatment method. The methods of early diagnosis of prostate cancer mainly include digital rectal examination, prostate needle biopsy, serum prostate-specific antigen (PSA) test, and magnetic resonance imaging (MRI) [5]. Digital rectal examination is affected by the experience levels of the doctor and is highly subjective; the serum PSA test lacks sufficient specificity; the needle biopsy shows higher false positive rate and invasive examination, and in most cases a second puncture is required $[6,7]$. MRI can well solve the above shortcomings and can be used to improve the clinical diagnosis of bone metastasis of prostate cancer.

With the development of MRI technology, it has been undertaken as the best choice for imaging diagnosis of prostate disease due to its advantages in high soft tissue resolution, no ionizing radiation and multiparameter imaging [8]. The detection, location, and staging of prostate cancer lesions rely on conventional MRI (T2WI), but it has certain limitations in the detection of central gland (CG) cancer, so its diagnostic specificity is low $[9,10]$. Therefore, a variety of techniques have emerged to improve the diagnostic capabilities of MRI for bone metastasis of prostate cancer, such as magnetic resonance elastic imaging (MRE), diffusion weighted imaging (DWI), and other functional MRI (fMRI) [11-13]. Different MRI techniques reflect different biological characteristics of prostate cancer, and the resulting images are also different. On DWI images, the signal of cancerous tissue is much higher than that of normal prostate tissue. Magnetic resonance spectroscopy (MRS) can detect the MR signal of multiple nuclei. China has a large population and a serious aging population, and limited medical resources are difficult to meet the growing social needs. Therefore, it has to choose a functional imaging technology that is quick and easy without reduction of diagnostic efficiency $[14,15]$, which should be convenient for clinical and effective MRI screening of bone metastasis of prostate cancer, aiming at early detection and early treatment of prostate cancer.

To explore the application of MRI in the diagnosis of bone metastasis of prostate cancer, 200 patients who were diagnosed with prostate cancer at hospital from February 23, 2020, to October 1, 2020, were selected as the research objects. They all performed MRI, DWI, and MRE. In addition, the bone metabolism indicators were detected before and after treatment for comparison to comprehensively evaluate the MRI images characteristics of prostate cancer with bone metastasis patients and the difference in the rehabilitation effect of patients with different bone metastasis conditions, aiming at providing a reference for the application value of multiple MRI techniques to detect the bone metastasis of prostate cancer.

\section{Materials and Methods}

2.1. Selection of Research Samples. 200 patients diagnosed with prostate cancer in hospital from February 23, 2017, to
October 1, 2020, were selected as the research objects, and they performed MRI, DWI, and MRE. The time interval between bone scan and MRI should not exceed 2 weeks. The patients were 55 to 85 years old, with an average age of $70 \pm 6.54$ years old.

The inclusion criteria were defined as follows: patients who were diagnosed as prostate cancer by three or more pathological studies; patients aged over 18 years; patients without relevant chemotherapy or drug treatment before surgery; and patients with clear consciousness and normal examination.

The exclusion criteria were determined as follows: patients with other malignant tumors, tumor metastasis, palliative surgery, and mental illness; and patients who withdrew from the experiment due to their own reasons.

2.2. Scanning Sequence and Parameters of MRI. Before the scan, the patient's renal function was examined to prevent the contrast agent from damaging the kidneys. Eating was prohibited 4-6 hours before the examination, and drinking water was allowed, aiming to ensure the cleanness of the rectum. When the signal was acquired from prostate MRI, the patient was required in a supine position. After the prostate anatomical positioning image was obtained, the patient was scanned with conventional axial MRI. The scan range of the prostate included bilateral seminal vesicle glands and prostate only. At least one scan range of the sequence had to cover the entire pelvis. After the scan, two MRI doctors (one of whom had 10 years of experience and the other had 12 years of experience) checked and blindly analyzed all MRI images without knowing the clinical diagnosis results.

The clinical criteria for diagnosing bone metastasis lesions were as follows. Abnormal radioactive concentration was visible by bone scan; bone metastasis lesion was confirmed by two or more influence line diagnosis methods, and the negative lesion confirmed by three or more imaging examinations was determined as NBM lesion; the record on puncture or surgical pathology history was available; one or more lesions were found and showed obvious development during the observation period; and the lesion area was reduced after radiotherapy.

2.3. Image Manifestations and Bone Metastasis Location Distribution. Among the 200 bone metastasis patients, a total of 156 patients were diagnosed by MRI, and the remaining 44 cases were false negatives. Bone metastasis lesions of 156 patients showed low signal under T2WI observation and showed nodular enhancement after enhancement. Under DWI observation, different degrees of patches or some nodular high signals were visible, and apparent diffusion coefficient (ADC) showed that the signal of the lesions was higher than that of the normal bone tissue. Among the 44 false-negative patients, 20 patients were diagnosed as MBM, with the T2WI signal in a mixed state and the uneven enhancement, and further imaging observations revealed that the low and high signals were mixed in the 
DWI and ADC images. 24 bone metastasis patients showed low signal on T2WI and enhanced signal after further enhancement, showing low signal in both DWI and ADC.

2.4. Observation Indicators. The patient's basic information (age, gender, height, and weight) and MRI images data were recorded. PSMA, PSA, and prostate volume (PV) of the patients were detected before and after the treatment. The changes of bone metabolism indicators $(\beta$-CTx, PINP, and BGP) were recorded and compared before and after treatment.

2.5. Statistical Methods. The data were processed and analyzed by SPSS19.0 version statistical software, the measurement data were expressed as mean \pm standard deviation $(\bar{x} \pm s)$, and the count data were displayed with percentage (\%). The sensitivity, specificity, and accuracy of MRI, DWI, and MRE on prostate cancer with bone metastasis were compared by independent $t$ test. PSMA, PSA, PV, $\beta$-CTx, PINP, and BGP in prostate cancer patients with osteogenic bone metastasis (OBM), mixed bone metastasis (MBM), and nonbone metastasis (NBM) were compared by analysis of variance. $P<0.05$ meant the difference was statistically significant.

\section{Results}

3.1. MRI Results of Some Patients. Figure 1 shows the MRI images of a male prostate cancer patient (aged 48 years old). It was clear that the prostate was enlarged, and nodular abnormal signal was visible in the right peripheral lobe of the prostate (with a size of about $2.4 \mathrm{~cm} \times 2.0 \mathrm{~cm}$ ). The T1 weighting showed a slightly low signal, the T2 weighting showed an uneven high signal, and the diffusion weighting showed a high signal. The lesion had not broken through the envelope, so failed to invade to the surroundings. No swollen lymph nodes were seen in the pelvis, and the pelvic bone signal was not abnormal.

Figure 2 shows the MRI images of a male prostate cancer patient (aged 60 years old). The prostate was enlarged and the pelvic wall was not thickened. After the enhanced scan, it showed a highly enhanced mucosal layer, and there was an unenhanced submucosa between the isoenhanced muscle layers.

\subsection{Bone Metastasis Diagnosis Results of Prostate Cancer} Patients. The bone metastasis diagnosis results of prostate cancer patients were illustrated in Figure 3. It revealed that there were $42 \mathrm{OBM}$ patients (21\%), $14 \mathrm{MBM}$ patients (7\%), and 144 NBM patients (72\%).

\subsection{Comparison on PSMA, PSA, and PV of Prostate Cancer} Patients with OBM, MBM, and NBM. The PSAs and PSMAs of prostate cancer patients with OBM, MBM, and NBM are compared in Figure 4 and 5, respectively. The PSA and PSMA of OBM patients were $202.15 \pm 31.53 \mathrm{ng} / \mathrm{mL}$ and $668.95 \pm 47.13 \mathrm{ng} / \mathrm{mL}$, respectively; those of MBM patients
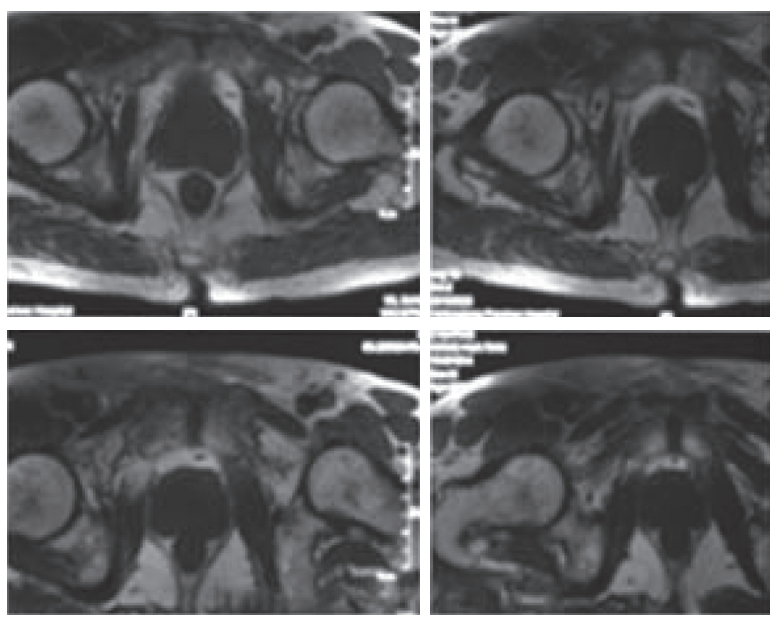

FIGURE 1: The MRI images of a male prostate cancer patient (aged 48 years old).
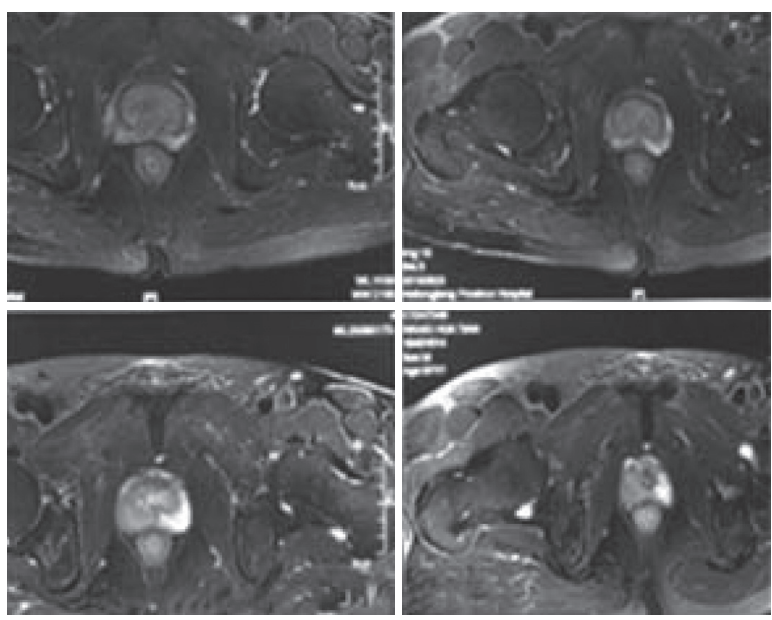

FIgURE 2: The MRI images of a male prostate cancer patient (aged 60 years old).

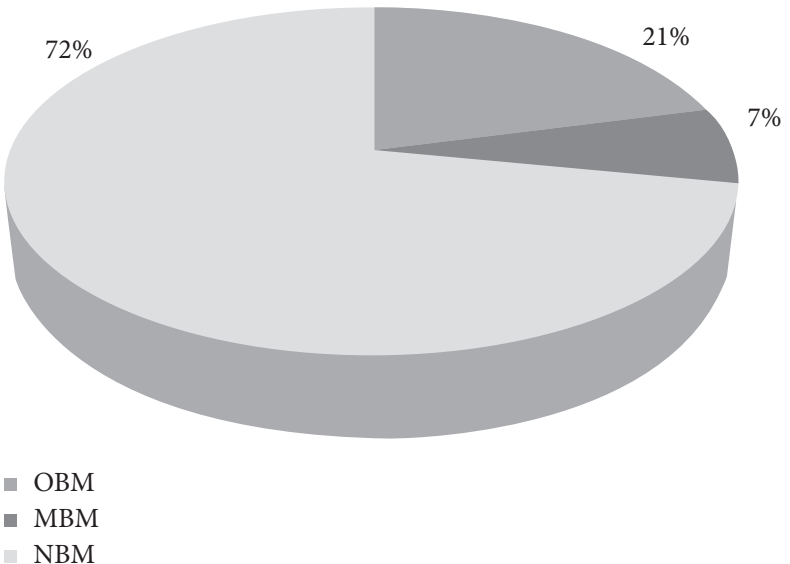

FIgURE 3: The bone metastasis diagnosis results of prostate cancer patients. 


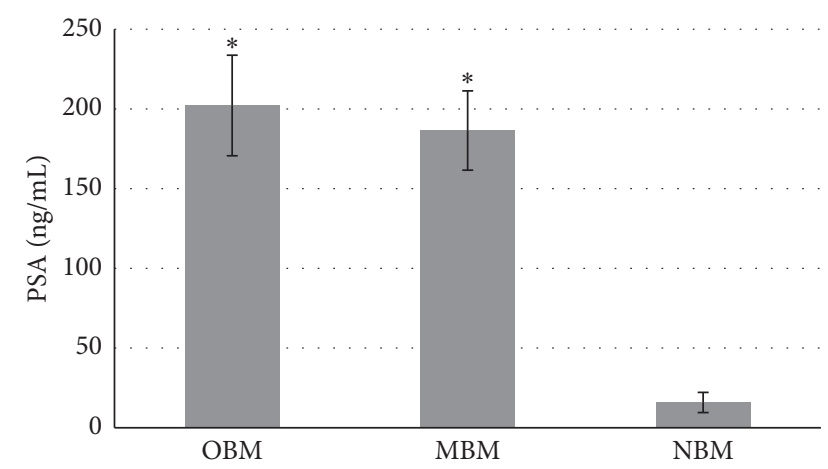

FIGURE 4: The comparison on PSAs of prostate cancer patients with OBM, MBM, and NBM. *Suggests that the difference was observable in contrast to the prostate cancer patients with NBM $(P<0.05)$.

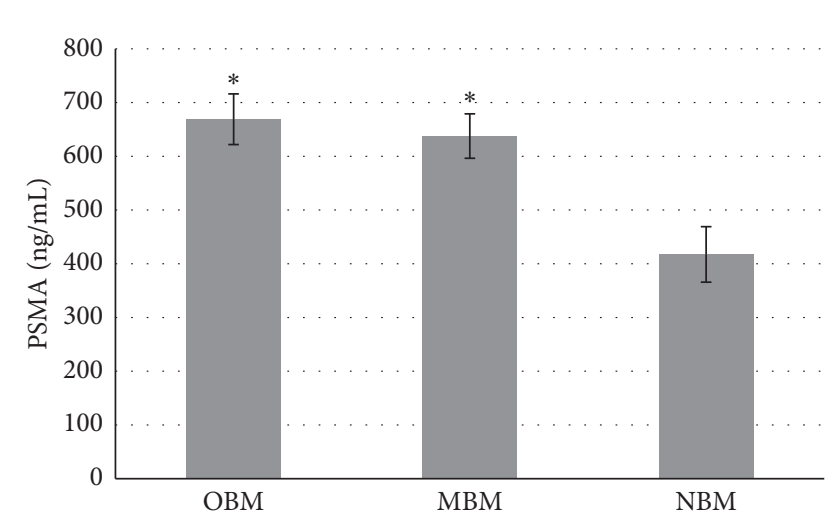

Figure 5: The comparison on PSMAs of prostate cancer patients with OBM, MBM, and NBM. *Suggests that the difference was observable in contrast to the prostate cancer patients with NBM $(P<0.05)$.

were $186.45 \pm 24.86 \mathrm{ng} / \mathrm{mL}$ and $637.63 \pm 41.35 \mathrm{ng} / \mathrm{mL}$, respectively; and those of NBM patients were $15.86 \pm 6.33 \mathrm{ng} /$ $\mathrm{mL}$ and $417.35 \pm 51.64 \mathrm{ng} / \mathrm{mL}$, respectively. Thus, PSMA and PSA of prostate cancer patients with OBM showed no statistical meaning with those of prostate cancer patients with MBM $(P>0.05)$, and the PSMA and PSA of prostate cancer patients with NBM showed obvious differences in contrast to those in the patients with OBM and $\mathrm{MBM}$ $(P<0.05)$.

As shown in Figure 6, the prostate volumes of prostate cancer patients with OBM, MBM, and NBM were compared. It showed that the prostate volumes of prostate cancer patients with OBM, MBM, and NBM were $68.45 \pm 8.33 \mathrm{~mL}$, $67.67 \pm 6.91 \mathrm{~mL}$, and $62.5 \pm 8.33 \mathrm{~mL}$, respectively. Thus, no visible difference could be found in prostate volumes among patients with OBM, MBM, and NBM.

3.4. Comparison on Detection Results of MRI, DWI, and MRE on Prostate Cancer Patients with Bone Metastasis. Figure 7 shows the comparison of sensitivity, specificity, and accuracy rate of combined detection of MRI, DWI, and MRE in the detection of prostate cancer with bone metastasis, respectively. The sensitivity, specificity, and accuracy rate of MRI for

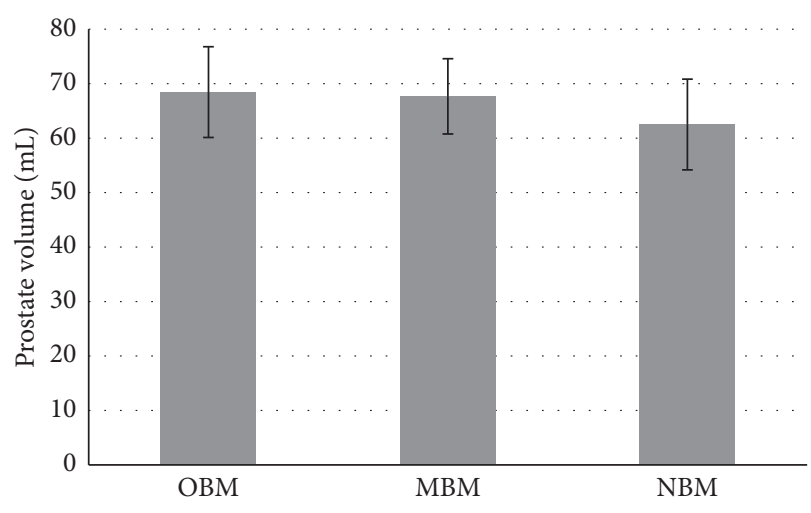

FIgURE 6: Comparison on prostate volume of prostate cancer patients with OBM, MBM, and NBM.

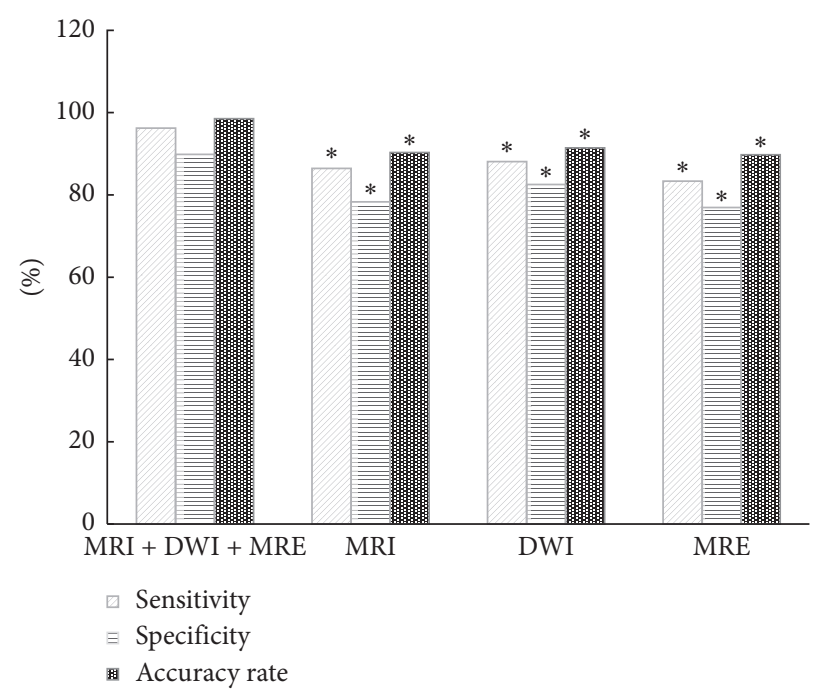

FIgURE 7: The comparison of sensitivity, specificity, and accuracy rate of combined detection of MRI, DWI, and MRE in the detection of prostate cancer with bone metastasis. ${ }^{*}$ Indicates that the difference was meaningful in contrast to the sensitivity of $\mathrm{MRI}+\mathrm{DWI}+\mathrm{MRE}(P<0.05)$.

detecting the prostate cancer with bone metastasis was $86.46 \%, 78.31 \%$, and $90.31 \%$, respectively; those of DWI was $88.11 \%, 82.53 \%$, and $91.43 \%$, respectively; those of MRE were $83.36 \%, 76.94 \%$, and $89.76 \%$, respectively; and those of MRI + DWI + MRE was 96.25\%, 89.85\%, and 98.53\%, respectively. Thus, the sensitivity, specificity, and accuracy rate of MRI + DWI + MRE were much higher in contrast of any single detection way of MRI, DWI, and MRE, showing statistical difference $(P<0.05)$, and there was no obvious difference in sensitivity, specificity, and accuracy rate of MRI, DWI, and MRE $(P>0.05)$.

3.5. Comparison on Bone Metabolism Indicators of Prostate Cancer Patients with OBM, MBM, and NBM before and after Treatment. Figure 8 illustrates the $\beta$-CTx levels of prostate cancer patients with OBM, MBM, and NBM before and after treatment. The $\beta$-CTx levels of prostate cancer patients with $\mathrm{OBM}$ before and after the treatment was $1.87 \pm 0.34 \mathrm{ng} / \mathrm{mL}$ 


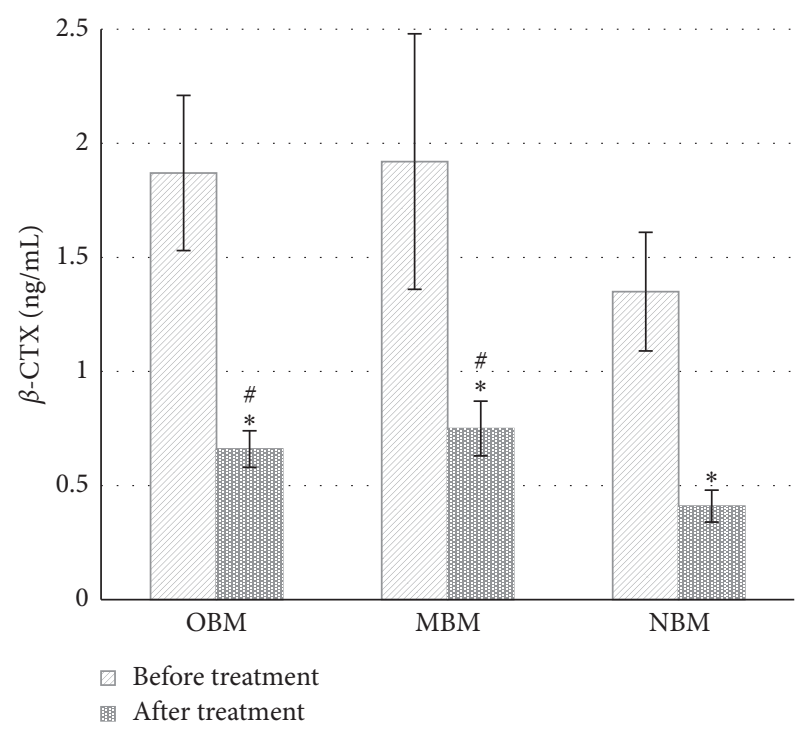

Figure 8: The $\beta$-CTx levels of prostate cancer patients with OBM, MBM, and NBM. * Suggests that the difference was remarkable in contrast to the levels before treatment $(P<0.05)$; and \# indicates dramatic difference could be found compared with the levels in patients with NBM after the treatment $(P<0.05)$.

and $0.66 \pm 0.08 \mathrm{ng} / \mathrm{mL}$, respectively; those of prostate cancer patients with MBM were $1.92 \pm 0.56 \mathrm{ng} / \mathrm{mL}$ and $0.75 \pm 0.12 \mathrm{ng} / \mathrm{mL}$, respectively; and those of prostate cancer patients with NBM were $1.35 \pm 0.25 \mathrm{ng} / \mathrm{mL}$ and $0.41 \pm 0.07 \mathrm{ng} / \mathrm{mL}$, respectively. Thus, it was clear that the $\beta$-CTx levels of all prostate cancer patients with different bone metastasis were decreased greatly after the treatment $(P<0.05)$. In addition, The $\beta$-CTX levels of patients with $\mathrm{OBM}$ and $\mathrm{MBM}$ prostate cancer after treatment were significantly lower than those of NBM patients, and the differences were statistically significant $(P<0.05)$.

Figure 9 illustrates the PINP levels of prostate cancer patients with OBM, MBM, and NBM before and after treatment. The PINP levels of prostate cancer patients with OBM before and after the treatment were $286.88 \pm 38.11 \mathrm{ng} /$ $\mathrm{mL}$ and $51.45 \pm 7.45 \mathrm{ng} / \mathrm{mL}$, respectively; those of prostate cancer patients with MBM were $265.27 \pm 30.22 \mathrm{ng} / \mathrm{mL}$ and $53.66 \pm 9.22 \mathrm{ng} / \mathrm{mL}$, respectively; and those of prostate cancer patients with NBM were $147.34 \pm 16.37 \mathrm{ng} / \mathrm{mL}$ and $39.04 \pm 6.38 \mathrm{ng} / \mathrm{mL}$, respectively. Thus, it was clear that the PINP levels of all prostate cancer patients with different bone metastases were decreased greatly after the treatment $(P<0.05)$ and that of prostate cancer patients with NBM was obviously lower in contrast to patients with the other two bone metastasis $s(P<0.05)$.

Figure 10 shows the levels of BGP before and after treatment in patients with OBM, MBM, and NBM prostate cancer. The BGP levels of patients with OBM prostate cancer before treatment was $86.45 \pm 10.31 \mathrm{ng} / \mathrm{mL}$, and the BGP level after treatment was $33.65 \pm 6.14 \mathrm{ng} / \mathrm{mL}$; the BGP level of patients with MBM prostate cancer before treatment was $78.56 \pm 8.94 \mathrm{ng} / \mathrm{mL}$, the BGP level after treatment was $31.24 \pm 5.73 \mathrm{ng} / \mathrm{mL}$; the BGP level of NBM prostate cancer patients before treatment was $54.23 \pm 5.86 \mathrm{ng} / \mathrm{mL}$;

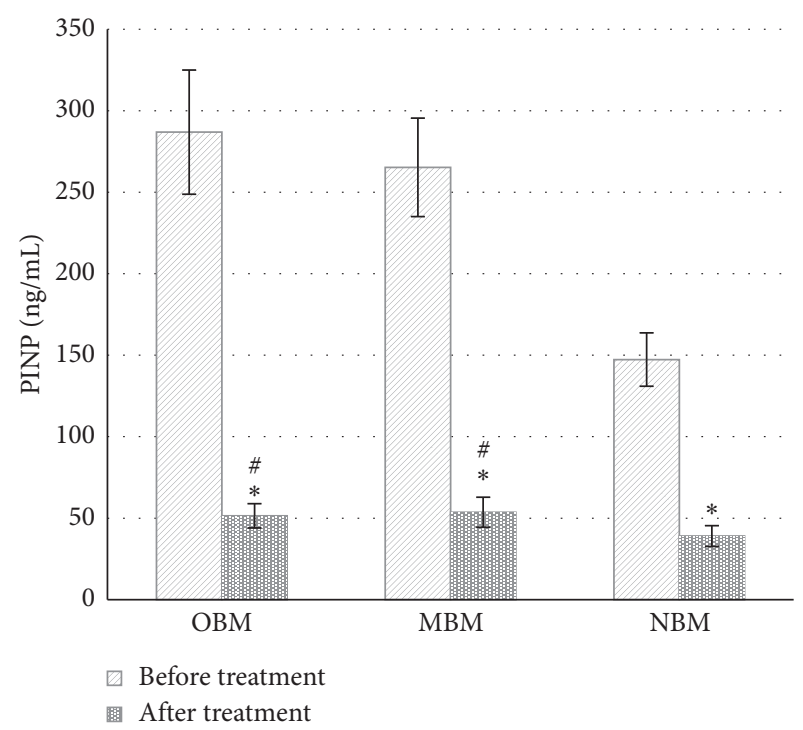

FIGURE 9: The PINP levels of prostate cancer patients with OBM, MBM, and NBM. *Suggests that the difference was remarkable in contrast to the levels before treatment $(P<0.05)$ and \# indicates dramatic difference could be found compared with the levels in patients with NBM after the treatment $(P<0.05)$.

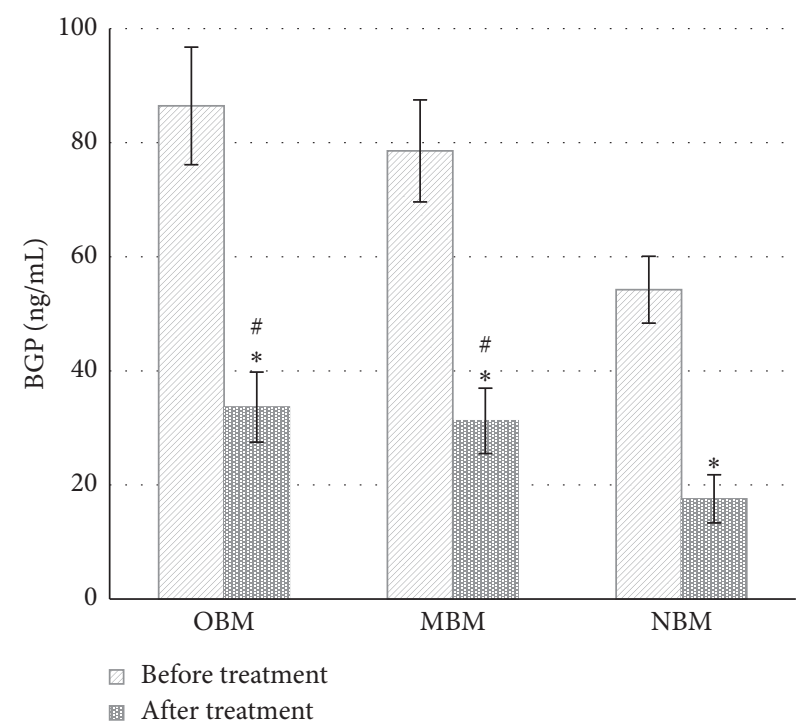

Figure 10: The BGP levels of prostate cancer patients with OBM, MBM, and NBM. *Suggests that the difference was remarkable in contrast to the levels before treatment $(P<0.05)$ and \# indicates dramatic difference could be found compared with the levels in patients with NBM after the treatment $(P<0.05)$.

and the BGP level after treatment was $17.56 \pm 4.22 \mathrm{ng} / \mathrm{mL}$. The BGP levels of prostate cancer patients with OBM, MBM, and NBM after treatment were obviously lower than those before treatment, showing remarkable differences $(P<0.05)$, and the BGP levels of prostate cancer patients with NBM after treatment were greatly lower in contrast to the levels of patients with the other two bone metastases, showing visible differences $(P<0.05)$. 


\section{Discussion}

Under physiological conditions, bone remodeling is mainly maintained by the interaction of osteoblasts and osteolytic cells. When tumor cells spread into the bone marrow, they will interfere with this interaction, and osteoblasts and osteolytic cells will be destroyed. The above is also considered to be the main mechanism of bone metastasis [16]. With the development of imaging technology and medical technology, clinical use of MRI to detect prostate cancer has become more and more widespread. In MRI images, prostate cancer signal changes will overlap with chronic prostatitis signals. Sometimes, chronic prostatitis and prostate cancer show similar manifestations under MRI observation, which may cause misdiagnosis. These two diseases have very similar MRI characteristics. In T2WI images, the lesions of these two diseases are characterized by low signal with multifocal and unclear borders. In addition, enhanced signal cannot completely distinguish the prostate cancer from chronic prostatitis, which has always revolved around urologists or radiologists [17]. Therefore, 200 patients who were diagnosed with prostate cancer in hospital from February 23, 2020, to October 1, 2020, were selected as the research objects and underwent MRI, DWI, and MRE. Their bone metabolism indicators were detected before and after treatment. The results found that there were $42 \mathrm{OBM}$ patients, 14 MBM patients, and 144 NBM patients, suggesting that the chance of prostate cancer with bone metastasis was not high, and the number of patients with OBM was higher than that of the MBM.

In clinical practice, prostate cancer with bone metastasis is very common. When a patient develops bone metastasis, it will be followed by complications such as bone pain, metastatic epidural spinal cord, and pathological fracture. These symptoms will seriously affect the quality of life of prostate cancer patients, shorten the patient's survival period, and bring economic burden to patients and families. In view of the above reasons, it has to find effective and targeted antibone metastasis treatments to relief the patients' pain, reduce complications, and prolong their lives [18]. At present, the curative effect evaluation standard of bone metastasis has not been formulated, and clinical trials are still needed to be extended to clinical treatment. The levels of PSMA4 $(17.35 \pm 51.64 \mathrm{ng} / \mathrm{mL})$ and PSA $(15.86 \pm 6.33 \mathrm{ng} / \mathrm{mL})$ in NBM prostate cancer patients were significantly lower than those of OBM $(668.95 \pm 47.13 \mathrm{ng} / \mathrm{mL}$ and $202.15 \pm 31.53 \mathrm{ng} / \mathrm{mL})$ and MBM $(637.63 \pm 41.35 \mathrm{ng} / \mathrm{mL}$ and $186.45 \pm 24.86 \mathrm{ng} / \mathrm{mL})$ prostate patients, showing statistically obvious differences $(P<0.05)$. Such results were similar to the results of von Hardenberg et al. [19], indicating that PSA and PSMA of prostate cancer patients with bone metastasis had been greatly increased compared with NBM patients. The sensitivity $(96.25 \%)$, specificity $(89.85 \%)$, and accuracy (98.53\%) of MRI + DWI + MRE in diagnosis of prostate cancer bone metastasis were observably higher than those of MRI $(86.46 \%, 78.31 \%$, and $90.31 \%)$, DWI $(88.11 \%$, $82.53 \%$, and $91.43 \%)$, and $\operatorname{MRE}(83.36 \%, 76.94 \%$, and $89.76 \%)$, showing statistically significant differences $(P<0.05)$. Such results indicated that combination of multiple MRI images could more effectively improve the diagnostic accuracy of prostate cancer with bone metastasis. The levels of $\beta$-CTX $(0.41 \pm 0.07 \mathrm{ng} / \mathrm{mL}), \quad$ PINP $(39.04 \pm 6.38 \mathrm{ng} / \mathrm{mL})$, and BGP $(17.56 \pm 4.22 \mathrm{ng} / \mathrm{mL})$ after treatment in patients with NBM prostate cancer were much lower than those of OBM $(0.66 \pm 0.08 \mathrm{ng} / \mathrm{mL}$, $51.45 \pm 7.45 \mathrm{ng} / \mathrm{mL}$, and $33.65 \pm 6.14 \mathrm{ng} / \mathrm{mL})$ and patients with MBM $(0.75 \pm 0.12 \mathrm{ng} / \mathrm{mL}, 53.66 \pm 9.22 \mathrm{ng} / \mathrm{mL}$, and $31.24 \pm 5.73 \mathrm{ng} / \mathrm{mL}$ ), showing statistically significant differences $(P<0.05)$. Such results were similar to the results of the study by Salembier et al. [20]. It suggested that the rehabilitation effect of NBM prostate cancer patients was more obvious and the treatment effect was better.

\section{Conclusion}

200 patients diagnosed with prostate cancer in hospital from February 23, 2020, to October 1, 2020, were selected as the research objects. All patients underwent MRI, DWI, and MRE, and their bone metabolism indicators were detected before and after the treatment. The results found that the sensitivity and specificity of MRI + DWI + MRE were better than those of any single detection method. For patients with suspected prostate cancer on bone scan, MRI could be performed to confirm the clinical stage of prostate cancer. Compared with NBM patients, the PSMA and PSA of bone metastasis prostate patients had been greatly improved, and there were great differences in the recovery effect in the later stage. However, there were still some shortcomings in this study. The selected samples were only prostate cancer patients, and no healthy volunteer controls were designed, lacking obvious contrast differences. In the follow-up, it will consider increasing the source of sample size and further explore the effects of MRI in diagnosis and treatment of prostate cancer patients with bone metastasis. In short, the results here could provide a theoretical basis for the application value of multiple MRI techniques in joint detection of prostate cancer patients with bone metastasis.

\section{Data Availability}

No data were used to support this study.

\section{Conflicts of Interest}

The authors declare that they have no conflicts of interest.

\section{References}

[1] D. Bonekamp, M. A. Jacobs, R. El-Khouli, D. Stoianovici, and K. J. Macura, "Advancements in MR imaging of the prostate: from diagnosis to interventions," Radiographics, vol. 31, no. 3, pp. 677-703, 2011.

[2] B. A. Mahal, J. M. Cohen, S. A. Allsop et al., "The role of phenotyping in chronic prostatitis/chronic pelvic pain syndrome," Current Urology Reports, vol. 12, no. 4, pp. 297-303, 2011.

[3] X. Zhuang, C. Chen, Z. Liu et al., "Multiparametric MRIbased radiomics analysis for the prediction of breast tumor regression patterns after neoadjuvant chemotherapy," 
Translational Oncology, vol. 13, no. 11, Article ID 100831, 2020.

[4] D. Volkin, B. Turkbey, A. N. Hoang et al., "Multiparametric magnetic resonance imaging (MRI) and subsequent MRI/ ultrasonography fusion-guided biopsy increase the detection of anteriorly located prostate cancers," BJU International, vol. 114, pp. E43-E49, 2014.

[5] H. Habchi, F. Bratan, A. Paye et al., "Value of prostate multiparametric magnetic resonance imaging for predicting biopsy results in first or repeat biopsy," Clinical Radiology, vol. 69, no. 3, pp. e120-e128, 2014.

[6] Y. Mazaheri, A. Shukla-Dave, D. A. Goldman et al., "Characterization of prostate cancer with MR spectroscopic imaging and diffusion-weighted imaging at 3Tesla," Magnetic Resonance Imaging, vol. 55, pp. 93-102, 2019.

[7] S. Fujii, T. Hayashi, Y. Honda et al., "Magnetic resonance imaging/transrectal ultrasonography fusion targeted prostate biopsy finds more significant prostate cancer in biopsy-naïve Japanese men compared with the standard biopsy," International Journal of Urology, vol. 27, no. 2, pp. 140-146, 2020.

[8] D. J. Winkel, H.-C. Breit, B. Shi, D. T. Boll, H.-H. Seifert, and C. Wetterauer, "Predicting clinically significant prostate cancer from quantitative image features including compressed sensing radial MRI of prostate perfusion using machine learning: comparison with PI-RADS v2 assessment scores," Quantitative Imaging in Medicine and Surgery, vol. 10 , no. 4 , pp. $808-823,2020$.

[9] J. O. Barentsz, J. Richenberg, R. Clements et al., "ESUR prostate MR guidelines 2012," European Radiology, vol. 22, no. 4, pp. 746-757, 2012.

[10] A. B. Rosenkrantz, H. Chandarana, J. Pfeuffer et al., "Zoomed echo-planar imaging using parallel transmission: impact on image quality of diffusion-weighted imaging of the prostate at 3T," Abdominal Imaging, vol. 40, no. 1, pp. 120-126, 2015.

[11] M. Pokorny, B. Kua, R. Esler et al., "MRI-guided in-bore biopsy for prostate cancer: what does the evidence say? A case series of 554 patients and a review of the current literature," World Journal of Urology, vol. 37, no. 7, pp. 1263-1279, 2019.

[12] O. Solyanik, B. Schlenker, C. Gratzke et al., "Bildgebung des lokal fortgeschrittenen Prostatakarzinoms: Die Bedeutung von Ultraschall und insbesondere MRT [Imaging of locally advanced prostate cancer: importance of ultrasound and especially MRI]," Der Urologe, vol. 56, no. 11, pp. 1383-1393, 2017.

[13] M. J. Watson, A. K. George, M. Maruf et al., "Risk stratification of prostate cancer: integrating multiparametric MRI, nomograms and biomarkers," Future Oncology, vol. 12, no. 21, pp. 2417-2430, 2016.

[14] M. Tenhunen, J. Korhonen, M. Kapanen et al., "MRI-only based radiation therapy of prostate cancer: workflow and early clinical experience," Acta Oncologica, vol. 57, no. 7, pp. 902-907, 2018.

[15] R. T. Gupta, B. Spilseth, N. Patel, A. F. Brown, and J. Yu, "Multiparametric prostate MRI: focus on T2-weighted imaging and role in staging of prostate cancer," Abdominal Radiology, vol. 41, no. 5, pp. 831-843, 2016.

[16] P. Dell'Oglio, A. Stabile, B. H. Dias et al., "Impact of multiparametric MRI and MRI-targeted biopsy on pre-therapeutic risk assessment in prostate cancer patients candidate for radical prostatectomy," World Journal of Urology, vol. 37, no. 2, pp. 221-234, 2019.

[17] L. Boesen, N. Nørgaard, V. Løgager et al., "Assessment of the diagnostic accuracy of biparametric magnetic resonance imaging for prostate cancer in biopsy-naive men: the biparametric mri for detection of prostate cancer (BIDOC) study," JAMA Network Open, vol. 1, no. 2, Article ID e180219, 2018.

[18] J. B. Gordetsky, J. V. Thomas, J. W. Nix, and S. Rais-Bahrami, "Higher prostate cancer grade groups are detected in patients undergoing multiparametric MRI-targeted biopsy compared with standard biopsy," American Journal of Surgical Pathology, vol. 41, no. 1, pp. 101-105, 2017.

[19] J. von Hardenberg, N. Westhoff, D. Baumunk et al., "Prostate cancer treatment by the latest focal HIFU device with MRI/ TRUS-fusion control biopsies: a prospective evaluation," Urology Oncology, vol. 36, no. 9, 2018.

[20] C. Salembier, G. Villeirs, B. De Bari et al., "ESTRO ACROP consensus guideline on CT- and MRI-based target volume delineation for primary radiation therapy of localized prostate cancer," Radiotherapy and Oncology, vol. 127, no. 1, pp. 49-61, 2018. 